\title{
BMJ Open A pragmatic randomised controlled trial of an implementation intervention to increase healthy eating and physical activity-promoting policies, and practices in centre-based childcare services: study protocol
}

\author{
Meghan Finch, ${ }^{1,2}$ Sze Lin Yoong, ${ }^{1,2}$ Rebecca J Thomson, ${ }^{1}$ Kirsty Seward, ${ }^{1,2}$ \\ Mairead Cooney, ${ }^{1}$ Jannah Jones, ${ }^{1,2}$ Alison Fielding, ${ }^{1}$ John Wiggers, ${ }^{1,2,3}$ \\ Karen Gillham, ${ }^{1}$ Luke Wolfenden ${ }^{1,2,3}$
}

To cite: Finch M, Yoong SL, Thomson RJ, et al. A pragmatic randomised controlled trial of an implementation intervention to increase healthy eating and physical activity-promoting policies, and practices in centre-based childcare services: study protocol. BMJ Open 2015;5:e006706. doi:10.1136/bmjopen-2014006706

\section{- Prepublication history and additional material is available. To view please visit the journal (http://dx.doi.org/ 10.1136/bmjopen-2014- 006706).}

Received 22 September 2014 Revised 1 April 2015 Accepted 10 April 2015

\section{CrossMark}

\footnotetext{
${ }^{1}$ Hunter New England Population Health, Newcastle, New South Wales, Australia ${ }^{2}$ School of Medicine and Public Health, University of Newcastle, Newcastle, New South Wales, Australia ${ }^{3}$ Hunter Medical Research Institute, Newcastle, New South Wales, Australia

Correspondence to Meghan Finch; meghan.finch@hnehealth. nsw.gov.au
}

\section{ABSTRACT}

Background: Promotion of healthy eating and physical activity in early childhood is recommended as a global chronic disease prevention strategy. Centrebased childcare services represent a promising setting to provide children with opportunities to improve healthy eating and physical activity. Evidence to inform implementation of childcare obesity prevention guidelines into routine practice in childcare, however, is lacking. This study aims to assess the effectiveness of an intervention, delivered to childcare staff, aiming to increasing service implementation of healthy eating and physical activity-promoting policies and practices.

Methods and analysis: A pragmatic parallel group randomised controlled trial will be undertaken with 165 childcare services in the Hunter New England region of New South Wales, Australia. Services will be randomised to receive either a 10-month evidencebased performance review intervention with other resources to support practice change, or to a waitlist control group. The primary trial outcome is the proportion of services implementing all of the following recommended healthy eating and physical activity promoting practices: written nutrition, physical activity and small screen recreation policies; providing information to families regarding healthy eating (including breastfeeding), physical activity and small screen time; providing twice weekly healthy eating learning experiences to children; providing water and only plain milk to children; providing fundamental movement skills activities for children every day; and limiting and using electronic screen time more for educational purposes and learning experiences. Effectiveness will be assessed using a telephone interview of practice implementation with childcare staff at baseline and 12 months following baseline.

Ethics and dissemination: The study was approved by the Hunter New England Human Research Ethics Committee and the University of Newcastle Human Research Ethics Committee. Study findings will be

\section{Strengths and limitations of this study}

The study has a strong design incorporating random allocation, pragmatic design and blinding of data collection interviewers.

- The intervention consists of a broad set of intervention strategies, is based on a theoretical framework and is of a long duration (10 months).

- A limitation of the trial is its reliance on the selfreport of service managers for the primary outcome measurement of the prevalence of service delivery policies and practices.

- The study will contribute to evidence regarding the effectiveness of an obesity prevention implementation intervention delivered in centre-based childcare settings.

disseminated widely through peer-reviewed publications and conference presentations.

Trial registration number: Australian New Zealand Clinical Trials Registry ACTRN12614000972628.

\section{BACKGROUND}

Globally, the prevalence of child overweight and obesity is increasing, with $9 \%$ of children aged $0-5$ years expected to be classified as overweight or obese by the year $2020 .{ }^{1}$ Childhood overweight and obesity tracks into adulthood, ${ }^{2}$ increasing the future health burden of a number of associated chronic health conditions including diabetes, cardiovascular disease and some cancers. ${ }^{3}{ }^{4}$ Inadequate physical activity and poor nutrition are key risk factors related to the development of overweight and obesity. ${ }^{5}$ Improving physical activity and diet in early childhood 
has been recommended as a strategy to mitigate the adverse public health impacts of excessive population weight gain. ${ }^{36}$

Centre-based childcare services have been identified as a key setting for the delivery of healthy eating and physical activity promoting interventions. ${ }^{7-10}$ Such services provide access to a large and growing number of children for prolonged periods of time each day, ${ }^{11-13}$ have existing infrastructure to facilitate healthy eating and physical activity for children, ${ }^{8}$ and are supported through licensing and accreditation standards to implement such practices. ${ }^{14}$ In Australia, healthy eating and physical activity best practice guidelines ${ }^{15}{ }^{16}$ recommend that childcare services have nutrition and physical activity policies; support consumption of healthy foods and drinks; educate children about healthy eating and support active play opportunities including development of movement skills; create positive mealtime environments; provide information to parents about healthy food choices and active play; and limit small screen recreation.

Despite such recommendations, evidence suggests these practices are not routinely implemented by childcare services. ${ }^{17-21}$ In Australia, for example, research has found that only $48-50 \%$ of services have a written physical activity policy and only $46-60 \%$ of programme time each day for fundamental movement skill sessions. ${ }^{20}$ Further, 25-28\% of Australian childcare services report providing opportunities each day for children to participate in sedentary screen time $\mathrm{e}^{20}$ and $60 \%$ of preschooler lunchboxes contain more than one serving of high fat, salt or sugar foods or drinks. ${ }^{21-23}$

The public health benefits of evidence-based healthy eating and physical activity practices recommended for childcare services will not benefit children unless they are implemented. ${ }^{24}$ Given the limited number of published obesity prevention implementation interventions conducted in childcare, there is a need for practice-relevant evidence to inform strategies that can effectively support adoption of healthy eating and physical activity policies and practices in this setting. ${ }^{19} 25$ This study will assess the effectiveness of a review and feedback intervention in increasing the implementation of healthy eating and physical activity-promoting policies and practices in childcare services. This paper describes the protocol for the primary trial outcome.

\section{METHODS}

\section{Study design}

The study will employ a pragmatic parallel group randomised controlled trial design with 165 childcare services. A sample of eligible childcare services, within the Hunter New England Region, New South Wales (NSW), Australia, will be randomly selected and approached to participate in the trial. Services will then be randomly allocated either to receive the intervention, delivered over a 10-month period, or to a wait list control group. Services allocated to the wait list control group will receive an intervention after the collection of all follow-up data. The primary trial outcome data will be collected via telephone surveys conducted at baseline and at follow-up. Findings from the study will be reported in accordance with the CONSORT statement. ${ }^{26}$

\section{Setting}

The study will be developed and evaluated as a pragmatic implementation trial conducted as part of an initiative by a population health service delivery unit (Hunter New England Population Health). The Hunter New England Region encompasses non-metropolitan 'major cities', 'inner regional', 'outer regional' and 'remote' areas, as described by the Australian Standard Geographic Classification system. ${ }^{27}$ There are 843321 people residing in the area of which 33343 are children aged $3-5$ years. ${ }^{28}$ Eight per cent of residents speak languages other than English and $4.6 \%$ of residents are of Aboriginal or Torres Strait Islander origin. ${ }^{28}$ The Hunter New England Region has lower indices of socioeconomic status than the NSW state average. ${ }^{27}$ There are a total of 366 preschools and long day care services in the study region. In the state of New South Wales, preschools provide centre-based care for $6-8 \mathrm{~h} /$ day and enrol children between 3 and 6 years. Long day care services in New South Wales provide centre-based care for eight or more hours per day for 5 days/week and usually enrol children from 6 weeks to under 6 years. ${ }^{29}$ Both types of services provide specific preschool programmes for children aged 3-5 years that aim to provide early educational activities to help children prepare for school. ${ }^{29}$

\section{Sample}

A list of all childcare services in the region provided by the Early Childhood Education and Care Directorate (the Government Licensing Authority) will serve as the sampling frame. Services catering solely for special needs populations, such as children with vision or hearing impairment, will be excluded from participating in the trial, given the specialist care required for such children and the likelihood of a differential effect of the intervention in this population group, and those participating in other intervention trials targeting children's diet or physical activity will be excluded. Furthermore, we will use local health service data to exclude services already identified as comprehensively implementing healthy eating and physical activity practices. Eligibility of the remaining services will be confirmed with the Nominated Supervisor (manager) of the service during phone contact as part of the recruitment process. Nominated Supervisors (service managers) at eligible services will be invited to provide consent for their service to participate in the data collection procedures of the study (see online supplementary appendix 1 for information statement).

\section{Random allocation of childcare services}

Childcare services eligible for the trial will be randomly allocated to the intervention or control wait list group in 
a 1:1 ratio using a computerised random number function in Microsoft Excel. Allocation of services will be undertaken by a statistician who will not be involved in recruitment, intervention delivery or data collection. The randomisation of services will not be stratified, given little evidence that service characteristics such as geographic or socioeconomic characteristics of the service location are associated with the trial outcomes. ${ }^{20}$ This will be an 'open' trial due to the difficulty in blinding services to their group allocation and, therefore, services will be aware of which group they are assigned to.

\section{Intervention}

\section{Intervention development}

The intervention will seek to increase service implementation of healthy eating and physical activity policies and practices, consistent with best practice Australian healthy eating and physical activity guidelines for the childcare setting, ${ }^{15}$ and evidence reviews of policies and practices shown to be associated with child healthy eating and physical activity. ${ }^{19} 30$ Targeted practices and policies are a subset of 15 practices for which data is routinely collected by the health service and which have been identified to have low implementation adherence. Development of the intervention content will be informed by Damschroder's Consolidated Framework for Implementation Research. ${ }^{31}$

The intervention will consist of five performance review and feedback cycles provided to the Nominated Supervisor of each service across a period of 10 months. The first review and feedback cycle will be performed face to face with Nominated Supervisors and take around one and a half hours. Subsequent review and feedback cycles will be conducted over the phone. Each feedback cycle will take approximately $30 \mathrm{~min}$ to complete and will include:

1. A standard assessment of current implementation of targeted policies and practices via completion by the Nominated Supervisors (service managers) of survey items administered by a support officer.

2. The provision of verbal feedback to Nominated Supervisors by Support Officers regarding current policy and practice implementation and progress toward: Nominated Supervisor establishment of goals (in consultation with support officers) to further improve or sustain implementation; discussion with support officers to facilitate problem solving to alleviate anticipated implementation barriers; and the development of an implementation action plan. A written feedback report regarding implementation progress will also be sent to the Nominated Supervisors following each review and feedback cycle.

During the first face-to-face contact, the Nominated Supervisors will be asked to indicate their support for implementation of the targeted practices by signing a 'registration' document outlining the expectations and responsibilities of service staff regarding implementation. Furthermore, throughout the intervention, Nominated
Supervisors will be encouraged to communicate feedback regarding progress towards the policies and practices, and associated actions plans, with other staff at relevant service meetings. ${ }^{32-34}$

Based on systematic reviews of empirical research, ${ }^{35} 36$ the intervention will incorporate the following components, shown to be associated with greater effect in audit and feedback interventions targeting professional practice change:

A. Feedback provided by a reputable source: Feedback will be provided by usual support officers with qualifications in nutrition, exercise physiology, psychology and health education, and with at least 2 years' experience working with childcare services. ${ }^{35}$

B. Provision of multimodal feedback: Performance feedback will be provided to services in person (at the initial visit), via telephone and through written feedback. ${ }^{35}$ The verbal feedback will be linked to clear, measurable goals and action plans to improve the identified practice.

C. Tailored feedback: All forms of feedback will be tailored to individual service needs, context and implementation barriers. During feedback, support officers will discuss findings of the review, identify where the implementation of policies and practices links with setting continuous quality improvement processes and standards, provide reinforcement when positive progress has been made, discuss identified deficits and facilitate reflection, problem solving, goal setting and action planning. ${ }^{32} 37-39$

D. Recognition: Services that demonstrate achievement of all healthy eating and physical activity policies and practices will receive a certificate of recognition, and will be promoted to other services through newsletters. ${ }^{32}$

Intervention services will have access to other hard copy and electronic resources such as policy templates, a DVD demonstrating each fundamental movement skill (FMS) skill for educators, lanyards to be worn by childcare service staff during outdoor play with pictures and instructions for demonstrating each FMS, templates and a manual to assist with programming age-appropriate healthy eating learning experiences, healthy food guideline posters and parent lunchbox resources.

All intervention services will receive quarterly interactive email newsletters, which will communicate key messages relating to the targeted healthy eating and physical activity policies and practices. ${ }^{40}$ The electronic newsletters will provide web links for additional information, and will case study individual services that have undertaken successful and innovative approaches to implementation. The newsletters will include content to assist services to overcome key implementation barriers reported by childcare staff. All resources will be reviewed by local Nominated Supervisors and childcare service staff not participating in the trial, to ensure that they are well presented, relevant, attractive ${ }^{32}$ and culturally appropriate for Aboriginal services, families and children. 


\section{Intervention delivery, personnel and monitoring}

Intervention delivery will be overseen by an experienced health promotion project manager and delivered by trained support officers with experience providing support to such services. All intervention support staff will participate in fortnightly meetings with peers and the project manager to facilitate learning, to improve performance and service adherence, and to maintain protocol adherence and standardise intervention delivery. ${ }^{41}$ Any modifications to the intervention protocol will be submitted for approval to the investigating team with amendments documented using version control.

\section{Control group}

Participating services randomised to the wait list control group will receive the electronic newsletter, but will not receive any other intervention component or resources, as part of the trial. Childcare service staff within these services will be free to implement any of the recommended practices outlined in the newsletter. Following the completion of the intervention period, all control services will be offered the intervention.

\section{Data collection procedures}

Baseline and follow-up data will be coordinated by an experienced evaluation manager, who is also a member of the investigative team. Data will be collected via computer-assisted telephone interview (CATI) surveys with the Nominated Supervisor (or other lead educator) conducted annually as part of routine service delivery. Similar surveys have been used previously by the research team to assess the policies and practices of childcare services. $^{20} 4243$ The CATI survey will take approximately $15-25 \mathrm{~min}$ to complete, with all data entered electronically into secure databases held at Hunter New England Population Health. CATI interviewers involved in data collection will be blind to group allocation, and participating services will be asked not to disclose their group allocation during telephone data collection. CATI interviewers will be provided with training to facilitate understanding and adherence to data collection protocols, and to practice the survey script. The survey will be administered at baseline and repeated 12 months later. To minimise attrition, prior to follow-up data collection, all participating services in intervention groups will receive letters thanking them for their participation to date and reminding them of the follow-up data collection. Only the investigative team will have access to the information obtained from the participants. All electronic data will be stored in confidential computer files, which will be password protected at Hunter New England Population Health.

\section{Measures}

Trial outcomes and measures

The primary outcome is the change in prevalence of services implementing all targeted healthy eating and physical activity policies and practices. A validated survey tool previously used in other studies with Australian childcare services $^{20} 4243$ will be used to assess healthy eating and physical activity policies and practices. ${ }^{44}$

Specifically, these policies and practices are:

1. Having a service policy (nutrition, physical activity and small screen recreation). ${ }^{30}$

2. Service providing information to families (healthy eating, physical activity, small screen time and breast feeding, where relevant) ${ }^{45}$

3. Service providing structured and specific learning experiences about healthy eating at least two times/ week. ${ }^{45}$

4. Service supplying age-appropriate drinks to children (ie, only water and age-appropriate milk). ${ }^{46}$

5. Service conducting fundamental movement skills activities for children aged 3-5 years every day to at least $90 \%$ of children. ${ }^{47}$

6. Service limiting use of small screen recreation by children aged 3-5 years to only educational purposes and for learning experiences. ${ }^{48}$

Twelve items will be used to assess the prevalence of implementation of each of the six policies and practices targeted by the intervention. Validation of the items against observations undertaken by trained observers were previously undertaken for nutrition policy ( $\kappa 0.5$ ), physical activity policy ( $\kappa 0.59$ ), small screen recreation policy $(\kappa 0.77)$, service provision of water $(\kappa 0.78)$ and age-appropriate milk ( $\kappa 0.88$ ), provision of fundamental movement skills activities for children aged 3-5 years every day $(\kappa 0.06)$ to at least $90 \%$ of children ( $\kappa 0.2$ ) and screen time recreation $(\kappa 0.17) .{ }^{44}$

\section{Data collection management and monitoring}

As this is not a clinical trial, and no serious safety issues attributable to study participation were considered possible, an independent data monitoring committee was not established for this study. Data quality assurance measures will be embedded during conduct of the CATI, including staff training to ensure standard administration of survey items and inclusion of range checks for numerical questions as appropriate (eg, number of staff in service, number of children enrolled). Weekly summaries of survey completion rates and reasons for noncompletion of survey will also be monitored. However, all components of the programme will be subject to review by the project team and committees within the programme governance structure. This will include regular reporting to a Programme Advisory Group. Monthly reviews based on the performance review data provided to the investigative team will be undertaken to ensure the research project remains valuable and is acceptable to participants. Meanwhile, no adverse events are anticipated.

Nominated Supervisors and staff will be provided a phone number of the Research Ethics Committee as an avenue for reporting adverse events, should they arise. The details of any reported adverse event received by research staff will be documented according to the 
HNEHREC guidelines and forwarded to the Hunter New England Human Research Ethics Committee by the investigative team as soon as possible. The investigative team will have the responsibility of discontinuing the trial based either on adverse events or acceptability. In such a case, participating services will be contacted and informed of the decision.

\section{Dissemination}

All Nominated Supervisors and parents will receive a summary report of the findings of the study, and the results of the survey for all services. The investigative team will approve the requests paper and abstracts produced using trial data according to predetermined publication priorities based on appropriateness and scientific merit. Authorship will be determined according to Hunter New England Population Health guidelines.

\section{Sample size and power calculations}

Assuming a $10 \%$ prevalence of control services implementing all targeted policies and practices at follow-up, 152 services (76 per group) will be sufficient to detect an absolute difference of $20 \%$ in the prevalence of services implementing all targeted policies or practices with $80 \%$ power and an $\alpha$ of 0.05 . Based on an attrition rate of approximately $10 \%,{ }^{49} 165$ services will need to be approached to obtain the desired sample at follow-up.

\section{Analysis}

All statistical analysis will be performed using SAS V.9.2 statistical software.

The primary trial outcome will be analysed under an intention to treat framework. Intervention effectiveness will be assessed using logistic regression models controlling for baseline value using all available data. Sensitivity analysis will be performed by imputing baseline observation for missing data at follow-up. ${ }^{50}$ An $\alpha$ value of 0.05 will be utilised to determine statistical significance. Subgroup analyses of the primary trial outcome will be reported by service geographic and socioeconomic classification. The Australian Standard Geographical Classification will be used to classify service postcodes as urban (regional cities and inner regional classifications) or rural areas. Postcodes ranked in the top 50\% of New South Wales, according to the Socioeconomic Indices for Areas, will be classified as 'higher socioeconomic services'. All statistical tests will be two tailed with an $\alpha$ of 0.05 .

\section{DISCUSSION}

This paper presents the design and rationale for a pragmatic randomised controlled trial of a multicomponent review and feedback intervention, to facilitate policy and practice implementation to promote healthy eating and physical activity by centre-based childcare services. This trial will advance the currently limited evidence in this field and contribute important information regarding the effectiveness of intervention strategies to facilitate the translation of best practice recommendations into routine practice of childcare services.

Strengths of this study include the trial's randomised, pragmatic design, the use of theoretical framework and broad set of intervention strategies, the long duration of the intervention ( 10 months), the implementation of procedures to reduce potential threats to internal validity, such as the blinding of data collection interviewers and computer-based randomisation of groups.

The study will contribute to evidence regarding the effectiveness of obesity prevention implementation interventions delivered under pragmatic conditions in centrebased childcare settings.

Contributors MF led the development of this manuscript and is responsible for protocol updates and revisions. LW, SLY, MF, RJT, MC and KG conceived the intervention concept. RJT, MC and KS delivered the intervention. LW, SLY, AF, JJ and JW contributed to the evaluation design and trial methodology and, together with MF, formed the investigative team. MF is responsible for communicating and documenting protocol amendments where the investigative team deems there has been substantive change to methods documented in this version. In such cases, MF will also be responsible for updating the trial registry. All authors read and approved the final version of this manuscript.

Funding This project was funded by the Hunter Medical Research Institute (HMRI) and receives infrastructure funding from Hunter New England Population Health and the University of Newcastle.

Competing interests None declared.

Ethics approval Ethical approval to conduct the study has been obtained from the Hunter New England Human Research Ethics Committee (approval number 06/07/26/4.04) and University of Newcastle Human Research Ethics Committee (approval number $\mathrm{H}-2008-0343$ ) Trial registration ACTRN12614000972628.

Provenance and peer review Not commissioned; externally peer reviewed.

Open Access This is an Open Access article distributed in accordance with the Creative Commons Attribution Non Commercial (CC BY-NC 4.0) license, which permits others to distribute, remix, adapt, build upon this work noncommercially, and license their derivative works on different terms, provided the original work is properly cited and the use is non-commercial. See: http:// creativecommons.org/licenses/by-nc/4.0/

\section{REFERENCES}

1. De Onis M, Blössner M, Borghi E. Global prevalence and trends of overweight and obesity among preschool children. Am J Clin Nutr 2010;92:1257-64.

2. Venn A, Thomson R, Schmidt $M$, et al. Overweight and obesity from childhood to adulthood: a follow-up of participants in the 1985 Australian Schools Health and Fitness Survey. Med J Austr 2007;186:458-60.

3. Australian Institute of Health and Welfare. Australia's health 2004. Canberra, 2004.

4. World Health Organisation. Interventions on Diet and Physical Activity. What works? Summary Report. WHO Press. 2009. 23 April 2014.

5. Candeias V, Armstrong T, Xuereb G. Diet and physical activity in schools: perspectives from the implementation of the WHO global strategy on diet, physical activity and health. Can J Public Health 2010;101:S28-30.

6. National Center for Chronic Disease Prevention and Health Promotion. Obesity: halting the epidemic by making health easier. Atlanta, 2011.

7. World Health Organisation. Population based approaches to childhood obesity prevention. Geneva, 2012.

8. Story M, Kaphingst KM, French $\mathrm{S}$. The role of child care settings in obesity prevention. Future Child 2006;16:143-68.

9. Goldfield G, Harvey A, Grattan K, et al. Physical activity promotion in the preschool years: a critical period to intervene. Int J Environ Res Public Health 2012;9:1326-42. 
10. Birch L, Ventura A. Preventing childhood obesity: what works? Int $J$ Obes (Lond) 2009;33:S74-81.

11. Mamedova S, Redford J. Early childhood program participation, from the National Household Education Surveys Program of 2012. Washington DC: National Center for Education Statistics, Institute of Education Sciences, U.S. Department of Education, 2013. 23 April 2014.

12. Huskinson T, Pye J, Median K, et al. Childcare and early years survey of parents 2011. UK Department for Education, 2013.

13. Australian Bureau of Statistics. Childhood Education and Care. http://www.abs.gov.au/AUSSTATS/abs@.nsf/Lookup/4402.0.55. 003Main+Features1June\%202011?OpenDocument

14. Australian Government Department of Education EaWR. Belonging, being \& becoming. The early years learning framework for Australia. ACT, 2009.

15. Australian Government Department of Health and Ageing. Get up and grow: healthy eating and physical activity for early childhood: director/coordinator book. Canberra, 2009.

16. NSW Ministry of Health. Munch and move resource manual: birth to five years. NSW, 2012.

17. Bellows L, Anderson J, Gould S, et al. Formative research and strategic development of a physical activity component to a social marketing campaign for obesity prevention in preschoolers. J Commun Health 2008;33:169.

18. Cashmore A, Jones S. Growing up active: a study into physical activity in long day care centers. J Res Child Educ 2008;23:179.

19. Larson N, Ward DS, Neelon SB, et al. What role can child-care settings play in obesity prevention? A review of the evidence and call for research efforts. J Am Diet Assoc 2011;111:1343-62.

20. Wolfenden L, Neve M, Farrell L, et al. Physical activity policies and practices of childcare centres in Australia. J Paediatr Child Health 2010;47:73-6.

21. Kelly B, Hardy L, Howlett S, et al. Opening up Australian preschoolers' lunchboxes. Aust N Z J Public Health 2010;34:288-92.

22. Maalouf J, Evers SC, Griffin M, et al. Assessment of mealtime environments and nutrition practices in child care centers in Georgia Child Obes 2014;9:437-45.

23. Local Authorities Coordinators of Regulatory Authorities. Nursery school nutrition survey report. London: Local Government Association, 2010.

24. Lombard CB, Harrison CL, Kozica SL, et al. Effectiveness and implementation of an obesity prevention intervention: the HeLP-her Rural cluster randomised control trial. BMC Public Health 2014; 14:608

25. Rabin BA, Glasgow RE, Kerner JF, et al. Dissemination and implementation research on community-based cancer prevention: a systematic review. Am J Prev Med 2010;38:443-56.

26. Moher D, Schulz KF, Altman DG. Group C. CONSORT 2010 statement: updated guidelines for reporting parallel group randomised trials. PLOS Med 2010;7:e1000251.

27. New South Wales Department of Health, Population Health Division The Health of the People of New South Wales-Report of the Chief Health Officer. Sydney, 2006.

28. Australian Bureau of Statistics. 2011 census of population health and housing. Canberra, 2012.

29. Australian Bureau of Statistics. Childhood education and care. CAT. 4402.0. Canberra, 2012.

30. McPherson ME, Homer CJ. Policies to support obesity prevention for children: a focus on of early childhood policies. Pediatr Clin North Am 2011;58:1521-41, xii.

31. Damschroder L, Aron D, Keith R, et al. Fostering implementation of health services research findings into practice: a consolidated framework for advancing implementation science. Implement Sci 2009;4:50.
32. Rohrbach LA, Grana R, Sussman S, et al. Type II translation: transporting prevention interventions from research to real-world settings. Eval Health Prof 2006;29:302-33.

33. Grol R, Wensing M. What drives change? Barriers to and incentives for achieving evidence-based practice. Med J Aust 2004;180:57-60.

34. Rohrbach LA, Graham JW, Hansen WB. Diffusion of a school-based substance abuse prevention program: predictors of program implementation. Prev Med 1993;22:237-60.

35. Ivers N, Jamtvedt G, Flottorp S, et al. Audit and feedback: effects on professional practice and healthcare outcomes. Cochrane Database Sys Rev 2012;6:CD000259.

36. Alvero AM, Bucklin BR, Austin J. An objective review of the effectiveness and essential characteristics of performance feedback in organizational settings (1985-1998). J Organ Behav Manag 2001;21:3-29.

37. Soumerai SB, Avorn J. Principles of educational outreach ('academic detailing') to improve clinical decision making. JAMA 1990;263:549-56.

38. Archer JC. State of the science in health professional education: effective feedback. Med Educ 2010;44:101-8.

39. Baker R, Camosso-Stefinovic J, Gillies C, et al. Tailored interventions to overcome identified barriers to change: effects on professional practice and health care outcomes. Cochrane Database Sys Rev 2010;(3):CD005470.

40. Noar S, Benac C, Harris M. Does tailoring matter? Meta-analytic review of tailored print health behaviour change interventions. Psychol Bull 2007;133:673-93.

41. Sanders MR, Cann W, Markie-Dadds C. The triple P-Positive Parenting Programme: a universal population-level approach to the prevention of child abuse. Child Abuse Rev 2003;12:155-71.

42. Bell AC, Davies L, Finch M, et al. An implementation intervention to encourage healthy eating in centre-based child-care services: impact of the Good for Kids. Good for Life programme. Public Health Nutr 2015;18:1610-9.

43. Finch $\mathrm{M}$, Wolfenden $\mathrm{L}$, Falkiner $\mathrm{M}$, et al. Impact of a population based intervention to increase the adoption of multiple physical activity practices in centre based childcare services: a quasi experimental, effectiveness study. Int J Behav Nutr Phys Act 2012;9:101.

44. Dodds $\mathrm{P}$, Wyse $\mathrm{R}$, Jones $\mathrm{J}$, et al. Validity of a measure to assess healthy eating and physical activity policies and practices in Australian childcare services. BMC Public Health 2014;14:572.

45. Larson N, Ward D, Neelon SB, et al. Robert Wood Johnson Foundation. Preventing obesity among preschool children: how can child-care settings promote healthy eating and physical activity? Research synthesis. Robert Wood Johnson Foundation, 2011.

46. National Health and Medical Research Council. Australian dietary guidelines. Canberra, 2013.

47. Logan SW, Robinson LE, Wilson AE, et al. Getting the fundamentals of movement: a meta-analysis of the effectiveness of motor skill interventions in children. Child Care Health Dev 2012;38:305-15.

48. Okley T, Salmon J, Vella S, et al. A systematic review to inform the Australian sedentary behaviour guidelines for children and young people. Report prepared for the Australian Government Department of Health. Canberra, 2012.

49. Jones $\mathrm{J}$, Wolfenden $\mathrm{L}$, Wyse $\mathrm{R}$, et al. A randomised controlled trial of an intervention to facilitate the implementation of healthy eating and physical activity policies and practices in childcare services. BMJ Open 2014;4:e005312.

50. White IR, Horton NJ, Carpenter J. Strategy for intention to treat analysis in randomised trials with missing outcome data. BMJ 2011;342:d40. 